\title{
THE PROBABILITY OF LARGE DEVIATIONS FOR THE SUM FUNCTIONS OF SPACINGS
}

\author{
SHERZOD MIRA'ZAM MIRAKHMEDOV
}

Received 16 March 2004; Revised 23 November 2005; Accepted 28 November 2005

Let $0=U_{0, n} \leq U_{1, n} \leq \cdots \leq U_{n-1, n} \leq U_{n, n}=1$ be an ordered sample from uniform $[0,1]$ distribution, and $D_{i n}=U_{i, n}-U_{i-1, n}, i=1,2, \ldots, n ; n=1,2, \ldots$, be their spacings, and let $f_{1 n}, \ldots, f_{n n}$ be a set of measurable functions. In this paper, the probabilities of the moderate and Cramer-type large deviation theorems for statistics $R_{n}(D)=f_{1 n}\left(n D_{1 n}\right)+\cdots+$ $f_{n n}\left(n D_{n n}\right)$ are proved. Application of these theorems for determination of the intermediate efficiencies of the tests based on $R_{n}(D)$-type statistic is presented here too.

Copyright (c) 2006 Hindawi Publishing Corporation. All rights reserved.

\section{Introduction}

Let $U_{1}, U_{2}, \ldots$ be a sequence of independent uniform $(0,1)$ random variables (r.v.). We denote the order of $U_{1}, U_{2}, \ldots, U_{n-1}$ by $U_{1 n} \leq \cdots \leq U_{n-1, n} \leq U_{n n}$. Putting $U_{0, n}=0$ and $U_{n, n}=1$, we define spacings as $D_{i n}=U_{i n}-U_{i-1, n}, i=1,2, \ldots, n ; n=1,2, \ldots$, and denote $D=\left(D_{1 n}, \ldots, D_{n n}\right)$. Let $f_{m}(y)=f_{m n}(y), m=1,2, \ldots, n$, be a measurable functions of nonnegative argument $y$. We consider statistics of type

$$
R_{n}(D)=\sum_{m=1}^{n} f_{m}\left(n D_{m n}\right), \quad n=1,2, \ldots
$$

Statistics of this form have wide application, for example, goodness of fit tests, testing the dispersive ordering, for estimation of unknown parameters, in the problems of random coverage of the circle. An extensive survey on the distribution theory of these statistics and its applications can be found in Pyke [13, 14] and Deheuvels [2], see also, Feller [4], L'Ecuyer [10], and Ghosh and Jammalamadaka [5], and references therein.

The optimal condition of the asymptotical normality and the lower estimation of the remainder in CLT, Mirakhmedov [11], and Edgworth-type asymptotical expansion, Does et al. [3], and the probability of "supper large" deviation result (i.e., of order $c \sqrt{n}$ ), Zhou and Jammalamadaka [16], have been obtained for r.v. (1.1). But the topics of interest to us here are not readily available in the literature. In the present paper, we will prove 
a probability of large deviation theorems in moderate zone (i.e., of order $c \sqrt{\ln n}$ ) and Cramer's zone (i.e., of order $o(\sqrt{n})$ ).

Many authors have considered spacings-based tests testing hypothesis whether a random sample comes from a specified distribution. Most papers are dedicated to Pitman's approach (see, e.g., Holst and Rao [6], Jammalamadaka et al. [8]). Also in Zhou and Jammalamadaka [16], Bahadur's asymptotic efficiency and in Bartoszewicz [1] Bahadur's and Hodjes-Lehman approximate efficiencies have been studied. In the present paper, the intermediate efficiencies due to Kallenberg [9], see also Ivchenko and Mirakhmedov [7], of the spacings-based tests are presented too.

Thus the results of the present paper are filling existing gaps in the investigation of the large deviation probabilities of the statistics of type (1.1) and efficiencies of the tests based on this statistics.

The method developed in the present paper is based on following property of the uniform spacings. Let $Y_{1}(\lambda), Y_{2}(\lambda), \ldots$ be a sequence of independent r.v.'s with common exponential distribution $\exp (\lambda)$, and let $Y(\lambda)=\left(Y_{1}(\lambda), \ldots, Y_{n}(\lambda)\right), S_{n}(\lambda)=Y_{1}(\lambda)+\cdots+$ $Y_{n}(\lambda)$. For arbitrary $\lambda>0$, there is a regular variant of the conditional distribution of the random vector $Y(\lambda)$ given $S_{n}(\lambda)=n$ such that $\mathfrak{J}(n D)=\mathfrak{J}\left(Y(\lambda) / S_{n}(\lambda)=n\right)$, where $\mathfrak{J}(X)$ denoted the distribution of the random vector $X$. This equality is known and usually used at $\lambda=1$ (see, Pyke [13], Holst and Rao [6], Does et al. [3]). Its validity for arbitrary $\lambda>0$ follows from the fact that $S_{n}(\lambda)$ is a sufficient statistic for parameter $\lambda$. Thus for arbitrary measurable function $L\left(x_{1}, \ldots, x_{n}\right)$ of nonnegative arguments and each $\lambda>$ 0 , we have $\mathfrak{J}\left(L\left(n D_{1 n}, \ldots, n D_{n n}\right)\right)=\mathfrak{J}\left(L\left(Y_{1}, \ldots, Y_{n}\right) / S_{n}(\lambda)=n\right)$, and if $\int_{-\infty}^{\infty} \mid E L\left(Y_{1}(\lambda), \ldots\right.$, $\left.Y_{n}(\lambda)\right) \exp \left\{i \tau S_{n}(\lambda)\right\} \mid d \tau<\infty$, then

$$
E L\left(n D_{1 n}, \ldots, n D_{n n}\right)=\frac{1}{2 \pi p_{n}(n, \lambda)} \int_{-\infty}^{\infty} E\left(L\left(Y_{1}(\lambda), \ldots, Y_{n}(\lambda)\right) \exp \left\{i \tau\left(S_{n}(\lambda)-n\right)\right\}\right) d \tau,
$$

where $p_{n}(z, \lambda)$ is the density function of r.v. $S_{n}(\lambda)$. We note that the proof of the Cramertype theorem (Theorem 2.2) rests on a special choice of the parameter $\lambda$, see Lemma 3.2.

Few words about notations. Many quantities like $g_{m}, \rho, \tilde{g}_{m}$ depend on $n$, however for notational convenience, we will suppress this suffix, except cases where it is essential. $C_{i}$ is a positive constant that may not be the same in the different expressions, $\varepsilon$ is an arbitrary small positive constant. All asymptotic expressions are considered as $n \rightarrow \infty$.

The organization of this paper is as follows. The probabilities of large deviation results are formulated in Section 2. A proof of the theorems of Section 2 is given in Section 3. The application of the theorems of Section 2 to the study of the intermediate efficiency of tests based on spacing statistics are presented in Section 4. Appendix contains proofs of auxiliary lemmas.

\section{Results}

For simplicity of notations below, we put $Y_{m}=Y_{m}(1), S_{n}=S_{n}(1)$. Also we let

$$
R_{n}(Y)=\sum_{m=1}^{n} f_{m}\left(Y_{m}\right), \quad \rho=\operatorname{corr}\left(R_{n}(Y), S_{n}\right),
$$




$$
\begin{gathered}
g_{m}(u)=f_{m}(u)-E f_{m}\left(Y_{m}\right)-(u-1) \rho \sqrt{\frac{\operatorname{Var} R_{n}(Y)}{n}}, \\
T_{n}(D)=\sum_{m=1}^{n} g_{m}\left(n D_{m}\right), \quad T_{n}(Y)=\sum_{m=1}^{n} g_{m}\left(Y_{m}\right) .
\end{gathered}
$$

Note that $\sigma_{n}^{2} \equiv \operatorname{Var} T_{n}(Y)=\left(1-\rho^{2}\right) \operatorname{Var} R_{n}(Y)$ and

$$
E T_{n}(Y)=0, \quad \operatorname{cov}\left(T_{n}(Y), S_{n}\right)=0 .
$$

It is obviously that $T_{n}(D)=R_{n}(D)-E R_{n}(Y)$. Let $P_{n}(x)=P\left(T_{n}(D)<x \sigma_{n}\right)$ and let $\Phi(x)$ be the standard normal distribution function.

Theorem 2.1. If

$$
\begin{gathered}
\underline{\lim } \frac{1}{n} \sigma_{n}^{2}>0, \\
\varlimsup \frac{1}{n} \sum_{m=1}^{n} E\left|g_{m}\left(Y_{m}\right)\right|^{2+\delta}<\infty,
\end{gathered}
$$

for some $\delta>0$. Then for all $x$ such that $0 \leq x \leq \sqrt{\delta \ln n}$,

$$
1-P_{n}(x)=(1-\Phi(x))(1+o(1)), \quad P_{n}(-x)=\Phi(-x)(1+o(1)) .
$$

Theorem 2.2. Let (2.3) be fulfilled and for some $H>0$,

$$
\chi_{m} \stackrel{\text { def }}{=} E \exp \left\{H\left|g_{m}\left(Y_{m}\right)\right|\right\} \leq C .
$$

Then for $x \geq 0$ and $x=o(\sqrt{n})$,

$$
\begin{aligned}
& 1-P_{n}(x)=(1-\Phi(x)) \exp \left\{\frac{x^{3}}{\sqrt{n}} L_{n}\left(\frac{x}{\sqrt{n}}\right)\right\}\left(1+O\left(\frac{x+1}{\sqrt{n}}\right)\right), \\
& P_{n}(-x)=\Phi(-x) \exp \left\{-\frac{x^{3}}{\sqrt{n}} L_{n}\left(-\frac{x}{\sqrt{n}}\right)\right\}\left(1+O\left(\frac{x+1}{\sqrt{n}}\right)\right),
\end{aligned}
$$

where $L_{n}(u)=\ell_{0 n}+\ell_{1 n} u+\cdots$ is a special Cramer-type power series. Particularly, putting $\hat{\sigma}_{n}^{2}=n^{-1} \sigma_{n}^{2}, q_{i j}=n^{-1} \sum_{m=1}^{n} q_{i j m}$,

$$
\ell_{0 n}=\frac{1}{n} \hat{\sigma}_{n}^{-3} q_{30}, \quad \ell_{1 n}=\frac{1}{24 \hat{\sigma}_{n}^{4}} q_{40}-\frac{1}{8 \hat{\sigma}_{n}^{6}} q_{30}^{2}-\frac{1}{8 \hat{\sigma}_{n}^{4}} q_{21}\left(q_{21}+2 \hat{\sigma}_{n}^{2}\right)-\frac{1}{8 \hat{\sigma}_{n}^{4} n} \sum_{m=1}^{n} q_{20 m}^{2} .
$$

\section{Proof of Theorems 2.1 and 2.2}

Proofs based on the Cramer's transform. If $E e^{H|Y|}<\infty$, for some $H>0$, then the r.v. $X$ with distribution function $P\{X<u\}=E(\exp \{h Y\} 1\{Y<u\}) / E \exp \{h Y\}$ is called the Cramer's transform with parameter $h$ of the r.v. $Y$, where $|h|<H$. For r.v. $Y$, we have

$$
P\{Y>u\}=E e^{h Y} E(\exp \{-h X\} 1\{X>u\}) .
$$


Proof of Theorem 2.1. We will prove first relation from (2.5). Second relation can be obtained from first by substituting $-g_{m}(u)$ instead of $g_{m}(u), m=1,2, \ldots$ Under conditions of the Theorem 2.1 from [11, Corollary 3], it follows that $\left|P_{n}(x)-\Phi(x)\right| \leq C_{1} n^{-\delta^{\prime} / 2}$, where $\delta^{\prime}=\min (1, \delta)$. Using this and well-known relation

$$
1-\Phi(x)=\frac{1}{\sqrt{2 \pi} x} e^{-x^{2} / 2}\left(1+O\left(\frac{1}{x}\right)\right)
$$

it is easy to check that Theorem 2.1 holds true for $0 \leq x \leq \sqrt{\delta^{\prime} \ln n / 2}$. From now on, we suppose that

$$
\sqrt{\frac{\delta^{\prime}}{2} \ln n} \leq x \leq \sqrt{\delta \ln n}
$$

We introduce the truncated functions $\tilde{g}_{m}(u)=g_{m}(u) 1\left\{\left|g_{m}(u)\right| \leq \varepsilon \sigma_{n}\right\}, m=1,2, \ldots$, where $\varepsilon>0$ will be chosen sufficiently small later on. Putting $\widetilde{T}_{m}(D)=\sum_{m=1}^{n} \widetilde{g}_{m}\left(n D_{m}\right)$, $\widetilde{P}_{n}(x)=P\left(\widetilde{T}_{n}(D)<x \sigma_{n}\right)$, we have

$$
\left|1-P_{n}(x)-(1-\Phi(x))\right| \leq\left|\left(1-\widetilde{P}_{n}(x)\right)-(1-\Phi(x))\right|+\left|P_{n}(x)-\widetilde{P}_{n}(x)\right| \equiv \nabla_{1}+\nabla_{2} .
$$

(i) Estimation of $\nabla_{1}$. For a complex variable $z$, we denote $\tilde{\varphi}_{n}(z)=E \exp \left\{z \tilde{T}_{n}(D)\right\}$. Let $\widetilde{G}_{n}$ be the Cramer's transform with parameter $h=x / \sigma_{n}$ of the r.v. $\widetilde{T}_{n}(D)$. Note that $\tilde{\varphi}_{n}(h) \geq 1 / 2$ for sufficiently large $n$. Estimation of the $\nabla_{1}$ rests on the following lemma, proof of which (being long) is given in the appendix.

LeMma 3.1. Under conditions of Theorem 2.1,

(1) $\sup _{u}\left|P\left\{\widetilde{G}_{n}^{*}<u\right\}-\Phi(u)\right|=O\left(n^{-\delta^{\prime} / 3\left(2+\delta^{\prime}\right)}\right)$, where $\widetilde{G}_{n}^{*}=\left(\widetilde{G}_{n}-x \sigma_{n}\right) / \sigma_{n}$;

(2) $\tilde{\varphi}_{n}(h)=\exp \left\{x^{2} / 2\right\}\left(1+O\left(n^{-\delta^{\prime} / 3\left(2+\delta^{\prime}\right)}\right)\right)$, where $\delta^{\prime}=\min (1, \delta)$.

Due to (3.1),

$$
\begin{aligned}
P\left(\widetilde{T}_{n}(D)>x \sigma_{n}\right)= & \widetilde{\varphi}_{n}(h) E\left[\exp \left\{-h \widetilde{G}_{n}\right\} 1\left\{\widetilde{G}_{n}>x \sigma_{n}\right\}\right] \\
= & \exp \left\{\ln \widetilde{\varphi}_{n}(h)-x^{2}\right\} E\left[\exp \left\{-h \widetilde{G}_{n}^{*}\right\} 1\left\{\tilde{G}_{n}^{*}>0\right\}\right] \\
=\exp \left\{\ln \tilde{\varphi}_{n}(h)-x^{2}\right\}[ & \int_{0}^{\infty} \exp \{-x u\} d \Phi(u) \\
& \left.+\int_{0}^{\infty} \exp \{-x u\} d\left(P\left\{\widetilde{G}_{n}^{*}<u\right\}-\Phi(u)\right)\right] .
\end{aligned}
$$

We denote the first and second summands inside the square brackets by $A_{1}$ and $A_{2}$, respectively. It can be readily shown that $A_{1}=\Phi(-x) \exp \left\{x^{2} / 2\right\}$. In $A_{2}$, first of all we integrate by part, and after this we use first assertion of Lemma 3.1 to get $A_{2}=O\left(n^{-\delta^{\prime} / 3\left(2+\delta^{\prime}\right)}\right)$. Use second assertion of Lemma 3.1 to get $\exp \left\{\ln \tilde{\varphi}_{n}(h)-x^{2}\right\}=\exp \left\{-x^{2} / 2\right\}(1+$ $\left.O\left(n^{-\delta^{\prime} / 3\left(2+\delta^{\prime}\right)}\right)\right)$. Now apply this and estimations of $A_{1}$ and $A_{2}$ in the equality (3.5) to 
get

$$
\begin{aligned}
& P\left(\widetilde{T}_{n}(D)>x \sigma_{n}\right) \\
& \quad=\exp \left\{-\frac{x^{2}}{2}\right\}\left(1+O\left(n^{-\delta^{\prime} / 3\left(2+\delta^{\prime}\right)}\right)\right)\left[\Phi(-x) \exp \left\{\frac{x^{2}}{2}\right\}+O\left(n^{-\delta^{\prime} / 3\left(2+\delta^{\prime}\right)}\right)\right] .
\end{aligned}
$$

Thus taking into account (3.2), we have

$$
\nabla_{1}=(1-\Phi(x)) O\left(x n^{-\delta^{\prime} / 3\left(2+\delta^{\prime}\right)}\right)
$$

(ii) Estimation of $\nabla_{2}$. Note that $p_{n}(n, 1)=n^{n}(n !)^{-1} e^{n}$, hence using Stirling's formula, we obtain

$$
\left|\frac{1}{\sqrt{2 \pi n} p_{n}(n, 1)}-1\right| \leq \frac{C}{n}
$$

We have

$$
\begin{aligned}
&\left\{\widetilde{T}_{n}(D)>u\right\}=\left\{T_{n}(D)>u, \bigcap_{m=1}^{n}\left\{\left|g_{m}\left(n D_{m n}\right)\right| \leq \varepsilon x \sigma_{n}\right\}\right\} \subseteq\left\{T_{n}(D)>u\right\}, \\
&\left\{T_{n}(D)>u\right\}=\left\{T_{n}(D)>u, \bigcap_{m=1}^{n}\left\{\left|g_{m}\left(n D_{m n}\right)\right| \leq \varepsilon x \sigma_{n}\right\} \cup\left\{T_{n}(D)>u, \bigcup_{m=1}^{n}\left\{\left|g_{m}\left(n D_{m n}\right)\right|>\varepsilon x \sigma_{n}\right\}\right\}\right. \\
&\left.\subseteq\left\{\widetilde{T}_{n}(D)>u\right\} \bigcup_{m=1}^{n}\left\{\left|g_{m}\left(n D_{m n}\right)\right|>\varepsilon x \sigma_{n}\right\}\right\} .
\end{aligned}
$$

Hence putting $X_{m}(u)=1\left\{\left|g_{m}(u)>\varepsilon x \sigma_{n}\right|\right\}$ and using formula (1.2), we get

$$
\begin{aligned}
\nabla_{2} & \leq \sum_{m=1}^{n} P\left\{\left|g_{m}\left(n D_{m n}\right)\right|>\varepsilon x \sigma_{n}\right\} \\
& =\sum_{m=1}^{n} E X_{m}\left(n D_{m n}\right) \leq \sum_{m=1}^{n} \frac{1}{2 \pi p_{n}(n, 1)} \int_{-\infty}^{\infty}\left|E\left(X_{m}\left(Y_{m}\right) \exp \left\{i \tau\left(S_{n}-n\right)\right\}\right)\right| d \tau \\
& \leq \sum_{m=1}^{n} \frac{E X_{m}\left(Y_{m}\right)}{2 \pi p_{n}(n, 1)} \int_{-\infty}^{\infty}\left|E \exp \left\{i \tau\left(S_{n}-Y_{m}\right\}\right)\right| d \tau
\end{aligned}
$$

Note that

$$
\left|E \exp \left\{i \tau Y_{k}\right\}\right|=\left(1+\tau^{2}\right)^{-1 / 2}
$$

Therefore, quite clear calculations show that the integral in the right-hand side of (3.10) does not exceed $\pi$. Thus taking into account (3.8) and using successively Chebishev-type 
6 Large deviations of the sum functions of spacings

inequality, condition (2.3), and relations (3.2) and (3.3) in (3.10), we get

$$
\begin{aligned}
\nabla_{2} & \leq C \sum_{m=1}^{n} E X_{m}\left(Y_{m}\right) \leq \frac{C}{\left(\varepsilon \cdot x \cdot \sigma_{n}\right)^{2+\delta}} \sum_{m}\left|g_{m}\left(Y_{m}\right)\right|^{2+\delta}=O\left(n^{-\delta / 2} x^{-(2+\delta)}\right) \\
& =(1-\Phi(x)) O\left(n^{-\delta / 2} x^{-(1+\delta)} \exp \left\{\frac{x^{2}}{2}\right\}\right) \\
& =(1-\Phi(x)) O\left((\ln n)^{-(1+\delta) \delta^{\prime} / 2}\right) .
\end{aligned}
$$

Substituting in (3.4) the estimates obtained for $\nabla_{1}$ and $\nabla_{2}$ in (3.7) and (3.12) yields Theorem 2.1.

Proof of Theorem 2.2. Let us prove the first relation and for $x>1$ only. The case $x<1$ is not of interest here. Second relation can be obtained from first by substituting $-g_{m}(u)$ instead of $g_{m}(u), m=1,2, \ldots$ For any $\lambda \in J_{\varepsilon}=(1-\varepsilon, 1+\varepsilon), \varepsilon>0$, and $|h| \leq H_{1}<\min (1-$ $\varepsilon, H) / 4$ using Holder's inequality and inequality $a^{j} \leq j ! \exp \{H|a|\} / H^{j}, a \in R, j \geq 0$, we have

$$
\begin{aligned}
E \mid g_{m} & \left(Y_{m}(\lambda)\right) \mid{ }^{j} Y_{m}^{i}(\lambda) \exp \left\{h\left|g_{m}\left(Y_{m}(\lambda)\right)\right|\right\} \\
& \leq \lambda E\left|g_{m}\left(Y_{m}\right)\right|{ }^{j} Y_{m}^{i} \exp \left\{h\left|g_{m}\left(Y_{m}\right)\right|+\varepsilon Y_{m}\right\} \\
& \leq \lambda i ! j ! H_{1}^{-(i+j)}\left(\frac{C_{0}}{1-2\left(H_{1}+\varepsilon\right)}\right)^{1 / 2} .
\end{aligned}
$$

Putting $\tilde{Y}_{m}(\lambda)=Y_{m}(\lambda)-1$, we introduce functions of the complex variables $u$ and $v$ :

$$
\begin{aligned}
K_{m}(u, v, \lambda) & =E \exp \left\{u g_{m}\left(Y_{m}(\lambda)\right)+v \tilde{Y}_{m}(\lambda)\right\} \\
& =\lambda \int_{0}^{\infty} \exp \{v(t-1)-\lambda t\} E \exp \left\{u g_{m}(t)\right\} d t, m=1, \ldots, n .
\end{aligned}
$$

We will consider an analytical continuity of the function $K_{m}(u, v, \lambda)$ as a function of the variable $\lambda$ into disk $J_{\varepsilon}=\{\lambda:|\lambda-1|<\varepsilon\}$ and we will use the same denotation for it. Put $S(H, \varepsilon)=\left\{(u, v, \lambda):|u| \leq H,|v| \leq H, \lambda \in J_{\varepsilon}\right\}$. By (3.13), the function $K_{m}(u, v, \lambda)$ is analytical in the region $S\left(H_{1}, \varepsilon\right)$ and moreover in $S\left(H_{2}, \varepsilon\right)$, where $H_{2}=H_{1} \sqrt{1-2\left(H_{1}+\varepsilon\right)} /$ $8 e^{H_{1}} \sqrt{C_{0}}$, we have

$$
\left|K_{m}(u, v, \lambda)-1\right| \leq E\left|u g_{m}\left(Y_{m}(\lambda)\right)+v Y_{m}(\lambda)\right| \exp \left\{H_{1}\left|g_{m}\left(Y_{m}(\lambda)\right)\right|+H_{1}\left|\tilde{Y}_{m}(\lambda)\right|\right\} \leq \frac{1}{2}
$$

Hence, also the function $\hat{K}_{m}(u, v, \lambda)=\ln K_{m}(u, v, \lambda)$ is analytic in the region $S\left(H_{2}, \varepsilon\right)$ and

$$
\left|\hat{K}_{m}(u, v, \lambda)\right| \leq \sum_{j=1}^{\infty} \frac{\left|1-K_{m}(u, v, \lambda)\right|^{j}}{j} \leq \ln 2,
$$

for each $m=1, \ldots, n$. 
Let $\left(\eta_{m}, \xi_{m}\right)$ be a random vector with distribution function (depending on real parameters $h$ and $\lambda$ ):

$$
P\left\{\eta_{m}<u, \xi_{m}<v\right\}=\frac{1}{K_{m}(h, 0, \lambda)} E \exp \left\{h g_{m}\left(Y_{m}(\lambda)\right)\right\} 1\left\{g_{m}\left(Y_{m}(\lambda)\right)<u, \tilde{Y}_{m}(\lambda)<v\right\} .
$$

Note that $\left(\eta_{1}, \xi_{1}\right),\left(\eta_{2}, \xi_{2}\right), \ldots$ is a sequence of independent random vectors. We denote

$$
\begin{aligned}
& \varphi_{n}(z)=E \exp \left\{z T_{n}(D)\right\}, \quad \eta=\sum_{m=1}^{n} \eta_{m}, \quad \xi=\sum_{m=1}^{n} \xi_{m}, \quad A(h, \lambda)=E \eta, \\
& \Lambda(h, \lambda)=E \xi, \sigma^{2}(h, \lambda)=D \eta, \\
& B^{2}(h, \lambda)=D \xi, \quad \rho(h, \lambda)=\operatorname{cov}(\eta, \xi), \mu^{2}(h, \lambda)=\sigma^{2}(h, \lambda)-\rho^{2}(h, \lambda) B^{-2}(h, \lambda) .
\end{aligned}
$$

Let $G_{n}$ be the Cramer's transform of the r.v. $T_{n}(D)$ then putting $G_{n}^{*}(h, \lambda)=\left(G_{n}-\right.$ $A(h, \lambda)) / \mu(h, \lambda)$ due to $(3.1)$, we get

$$
\begin{aligned}
P\left\{T_{n}(D)>x \sigma_{n}\right\}= & \exp \left\{\ln \varphi_{n}(h)-h A(h, \lambda)\right\} \\
& \cdot E\left[\exp \left\{-h \mu(h, \lambda) G_{n}^{*}(h, \lambda)\right\} 1\left\{G_{n}^{*}(h, \lambda)>\frac{x \sigma_{n}-h A(h, \lambda)}{\mu(h, \lambda)}\right\}\right] .
\end{aligned}
$$

We will choose $\lambda$ and $h$ according to Lemmas 3.2 and 3.3.

LEMMA 3.2. Under conditions of Theorem 2.2, there exists a unique solution of the equation

$$
\Lambda(h, \lambda)=0
$$

This solution can be represented as the power series

$$
\lambda(h)=\sum_{j=0}^{\infty} \lambda_{j n} h^{j}
$$

which is convergent for $|h| \leq H_{0}$, where $H_{0}>0$ does not depend on $n$ and

$$
\left|\lambda_{\text {in }}\right| \leq \frac{(1+\varepsilon)}{H_{0}^{i}}, \quad i=1,2, \ldots
$$

In particular, $\lambda_{0 n}=1, \lambda_{1 n}=0, \lambda_{2 n}=n^{-1} \sum_{m=1}^{n} E\left(Y_{m}-1\right) g_{m}^{2}\left(Y_{m}\right)$.

Proof. Function $\bar{\Lambda}(h, \lambda) \equiv(1 / n) \Lambda(h, \lambda)=\left.(1 / n) \sum_{m}(\partial / \partial v) \hat{K}_{m}(u, v, \lambda)\right|_{u=h, v=0}$ is analytic in the region $S\left(H_{2}, \varepsilon\right)$. Also by (3.16) and Cauchy's inequality, $|\bar{\Lambda}(h, \lambda)| \leq \ln 2 / H_{2}$. Hence in the region $S\left(H_{2}, \varepsilon\right)$, the function $\bar{\Lambda}(h, \lambda)$ is bounded uniformly with respect to $h, \lambda, n$, and 
it can be represented as power series with respect to variables $h$ and $\lambda-1$. This series is convergent uniformly with respect to $n$. It can be easily checked that $\bar{\Lambda}(0,1)=0$ and $(\partial / \partial \lambda) \bar{\Lambda}(h, \lambda)_{h=0, \lambda=1}=-1$. Therefore Lemma 3.2 follows from well-known theorem on series solution of an equation. We can readily check that $H_{0}=\varepsilon \sqrt{H_{2}} / 4(\varepsilon+2 \ln 2)^{2}$. The inequalities (3.22) follow from Cauchy's inequality for coefficient of the power series since the root $\lambda(h)$ is analytic at zero.

Lemma 3.3. Let $\lambda(h)$ be the root of (3.20). Under conditions of Theorem 2.2, there exists a unique real solution of the equation

$$
A(h, \lambda(h))=x \sigma_{n}
$$

This solution can be represented as the power series

$$
h=\sum_{j=1}^{\infty} h_{j n}\left(\frac{x}{\sigma_{n}}\right)^{j}
$$

where $h_{1 n}=1$, and there is $H_{0}>0$ such that

$$
\left|h_{\text {in }}\right| \leq \frac{C}{H_{0}^{i}}, \quad i=1,2, \ldots
$$

Proof. Putting $A(h)=n^{-1} A(h, \lambda(h))$, we rewrite (3.23) as $A(h)=n^{-1} x \sigma_{n}$. Obviously,

$$
A(h)=\left.\frac{1}{n} \sum_{m=1}^{n} \frac{\partial}{\partial u} \hat{K}_{m}(u, 0, \lambda)\right|_{u=h, \lambda=\lambda(h)}
$$

Therefore, $A(h)$ is an analytic function in the region $|h| \leq H_{2}$, and $|A(h)| \leq \ln 2 / H_{2}$ (by (3.16) and Cauchy's inequality), $A(0)=0$, and its derivative at point $h=0$ equals $n^{-1} \sigma_{n}^{2}>0$ (by (2.3)). Thus Lemma 3.3 follows from the theorem on inversion of the analytic functions.

In what follows, $h$ and $\lambda$ are roots of (3.20) and (3.23). It is evident that for each $i$ and $j$, the function $Q_{m}^{(i, j)}(u, v, \lambda) \stackrel{\text { def }}{=} \partial^{i+j} \hat{K}_{m}(u, v, \lambda) / \partial u^{i} \partial v^{j}$ is analytic in the region $S\left(H_{2}, \varepsilon\right)$, and hence can be represented as power series in a neighborhood of the point $(0,0,1)$. Due to (3.16), (3.21), (3.22), and Cauchy's inequality, we have for each $i$ and $j$,

$$
Q_{m}^{(i, j)}(h, 0, \lambda)=\sum_{l=1}^{\infty} q_{l m n}^{(i, j)} h^{l}=\sum_{l=1}^{k} q_{l m n}^{(i, j)} h^{l}+O\left(h^{k+1}\right), \quad\left|q_{l m n}^{(i, j)}\right| \leq C\left(H_{2}, H_{0}, \varepsilon\right)
$$


From (3.27) taking into account (2.2), we get

$$
\begin{gathered}
A(h, \lambda(h))=\sum_{m=1}^{n} Q_{m}^{(1,0)}(h, 0, \lambda)=\sigma_{n}^{2} h+O\left(n h^{2}\right)=\sigma_{n}^{2} h+O\left(n h^{2}\right), \\
\Lambda(h, \lambda(h))=\sum_{m=1}^{n} Q_{m}^{(0,1)}(h, 0, \lambda)=\frac{1}{2} \sum_{m=1}^{n} E g_{m}^{2}\left(Y_{m}\right)\left(Y_{m}-1\right)+O\left(n h^{3}\right), \\
B^{2}(h, \lambda(h))=\sum_{m=1}^{n} Q_{m}^{(0,2)}(h, 0, \lambda)=n+h \sum_{m=1}^{n} E g_{m}\left(Y_{m}\right)\left(Y_{m}-1\right)^{2}+O\left(n h^{2}\right)=n(1+O(h)), \\
\sigma^{2}(h, \lambda(h))=\sum_{m=1}^{n} Q_{m}^{(2,0)}(h, 0, \lambda)=\sigma_{n}^{2}+h \sum_{m=1}^{n} E g_{m}^{3}\left(Y_{m}\right)+O\left(n h^{2}\right)=\sigma_{n}^{2}(1+O(h)), \\
\rho(h, \lambda(h))=\sum_{m=1}^{n} Q_{m}^{(1,1)}(h, 0, \lambda)=h \sum_{m=1}^{n} E g_{m}^{2}\left(Y_{m}\right)\left(Y_{m}-1\right)+O\left(n h^{2}\right),
\end{gathered}
$$

It follows from (3.30), (3.31), and (3.32) that

$$
\mu^{2}(h, \lambda)=\sigma_{n}^{2}\left(1+O\left(h^{2}\right)\right)
$$

By (3.27), we have

$$
\ln K_{m}(h, 0, \lambda(h))=\sum_{l=2}^{\infty} k_{l m n} h^{l},
$$

where $k_{2 m n}=E g_{m}^{2}\left(Y_{m}\right) / 2$ and by Cauchy's inequality,

$$
\left|k_{i m n}\right| \leq \frac{3}{2 H_{0}^{i}}, \quad i=1,2, \ldots, m=1,2, \ldots, n .
$$

Future proof rests on Lemma 3.4, which will be proved in the appendix.

Lemma 3.4. Let $\lambda$ and $h$ be the roots of (3.20) and (3.23), respectively, and the conditions of Theorem 2.2 are fulfilled. Then

(1) $\sup _{u}\left|P\left\{G_{n}^{*}(h, \lambda)<u\right\}-\Phi(u)\right|=O(1 / \sqrt{n})$.

(2) $\varphi_{n}(h)=\left(\sqrt{2 \pi n} p_{n}(n, \lambda)\right)^{-1} \prod_{m=1}^{n} K_{m}(h, 0, \lambda)(1+O(h))$.

From (3.19) using first assertion of Lemma 3.4,

$$
\begin{aligned}
1-P_{n}( & x) \\
= & \exp \left\{\ln \varphi_{n}(h)-h A(h, \lambda(h))\right\} \\
& \times\left[\int_{0}^{\infty} \exp \{-h \sigma(h, \lambda(h)) v\} d \Phi(v)+\int_{0}^{\infty} \exp \{-h \sigma(h, \lambda(h)) v\} d\left(P\left\{G_{n}^{*}<v\right\}-\Phi(v)\right)\right] \\
= & \exp \left\{\ln \varphi_{n}(h)-h A(h, \lambda(h))\right\}\left[\exp \left\{h^{2} \sigma^{2}(h, \lambda(h)) / 2\right\} \Phi(-h \sigma(h, \lambda(h)))\right]+O\left(n^{-1 / 2}\right) .
\end{aligned}
$$


Also, $\exp \left\{h^{2} \sigma^{2}(h, \lambda(h)) / 2\right\} \Phi(-h \sigma(h, \lambda(h)))=(2 \pi)^{-1 / 2} M(h \sigma(h, \lambda(h)))$, where $M(u)=$ $\Phi(-u) / \Phi^{\prime}(u)$ is the ratio of Mills. Taking into account (3.31) and that $h=x \sigma_{n}^{-1}(1+$ $\left.O\left(x n^{-1 / 2}\right)\right)$ by standard manipulations, (see, Petrov [12]) $M(h \sigma(h, \lambda(h)))=M(x)(1+$ $\left.O\left(x n^{-1 / 2}\right)\right)$. Substitute this expression in (3.36) and take into account that $M(x) \geq x^{-1}$ to get

$$
1-P_{n}(x)=\exp \left\{\ln \varphi_{n}(h)-h A(h, \lambda(h))+x^{2} / 2\right\}(1-\Phi(x))\left(1+O\left(x n^{-1 / 2}\right)\right) .
$$

Since $p_{n}(z, \lambda)=\lambda^{n} z^{n-1} e^{-\lambda n} /(n-1)$ ! using Stirling's formula and Lemma 3.2,

$$
\begin{aligned}
\sqrt{2 \pi n} p_{n}(n, \lambda) & =\lambda^{n} e^{n(1-\lambda)}\left(1+O\left(n^{-1}\right)\right)=\exp \{n \ln (1-(1-\lambda))+n(1-\lambda)\} \\
& =\exp \left\{n \sum_{k=4}^{\infty} p_{k n} h^{k}\right\},
\end{aligned}
$$

where $\left|p_{k n}\right| \leq C\left(\varepsilon, H_{0}\right)$. From this relation and second assertion of Lemma 3.4 and (3.34), we get

$$
\frac{1}{n} \ln \varphi_{n}(h)=\frac{1}{n} \sum_{m=1}^{n} \ln K_{m}(h, 0, \lambda(h))-\frac{1}{n} \ln \left(\sqrt{2 \pi n} p_{n}(n, \lambda)\right)+O(h)=\sum_{j=2}^{\infty} \varphi_{j n} h^{j}+O(h),
$$

where $\left|\varphi_{j n}\right| \leq C\left(\varepsilon, H_{0}\right), j=2,3, \ldots$ with $\varphi_{2 n}=\sigma_{n}^{2} / 2 n$.

Due to (3.26) and (3.34), we have

$$
\frac{1}{n} A(h, \lambda(h))=\sum_{l=1}^{\infty} a_{m n} h^{l}, \quad\left|a_{i n}\right|<C\left(H_{0}, H_{2}, \varepsilon\right), \quad j=1,2, \ldots
$$

with $a_{1 n}=\sigma_{n}^{2} / n$.

From relations (3.39) and (3.40), substituting instead of $h$ its expression from (3.32), and collecting coefficients at powers of $x / \sqrt{n}$,

$$
\ln \varphi_{n}(h)-h A(h, \lambda(h))+\frac{x^{2}}{2}=n \sum_{l=3}^{\infty}\left(\varphi_{j n}-a_{j-1, n}\right) h^{l}+O(h)=\frac{x^{3}}{\sqrt{n}} L_{n}\left(\frac{x}{\sqrt{n}}\right)+O(h) .
$$

From (3.25), (3.35), (3.31), and (3.40), it follows that the series $L_{n}(u)$ are majorized by the power series with coefficient not depending on $n$. Theorem 2.2 follows now from (3.37) and (3.41).

\section{Asymptotical relative indeterminate efficiency}

Let $X_{1 n}, X_{2 n}, \ldots, X_{n-1, n}$ be an ordered sample from distribution $F(x)$, and $W_{k n}=X_{k n}-$ $X_{k-1, n}, k=1, \ldots, n$, with notations $X_{o n}=0$ and $X_{n n}=1$. We wish to test null hypothesis $H_{0}: F(x)=x, 0 \leq x \leq 1$, versus sequence of alternatives

$$
H_{1 n}: F_{n}(x)=x+L(x) \delta(n), \quad 0 \leq x \leq 1,
$$


where $\delta(n) \rightarrow 0$, as $n \rightarrow \infty$, and function $L(x)$ satisfy smoothness conditions, under which $W_{k n}$ can be related to the uniform spacings $D_{k n}$ by the relation (cf. $\left.[6,(3.2)]\right) n W_{k n}=$ $n\left[F_{n}^{-1}\left(U_{k n}\right)-F_{n}^{-1}\left(U_{k-1, n}\right)\right]=n D_{k n}\left(1-l\left(r_{k n}\right) \delta(n)+o_{p}(\delta(n))\right)$, where $l(u)=L^{\prime}(u), r_{k n}=$ $(k-0.5) / n$, and $o_{p}(\cdot)$ is uniform in $k$. Hence, Theorems 2.1 and 2.2 can be applied for test statistics of type

$$
R_{n}=\sum_{k=1}^{n} f\left(n W_{k n}, r_{k n}\right)
$$

We assume that function $f(u, y)$ is defined on $[0, \infty] \times[0,1]$ and has continuous derivatives of first and second orders with respect to $u$. Statistic $R_{n}$ is called symmetric if function $f(y, u)=f(y)$ does not depend on $u$, in otherwise statistic $R_{n}$ is nonsymmetric. A test based on statistic $R_{n}$ is called $f$-test.

We will apply Theorems 2.1 and 2.2 to the analysis of the intermediate asymptotic efficiency (IAE) due to Kallenberg [9] (see, also Ivchenko and Mirakhmedov [7]) of the $f$-tests. Note that asymptotical properties of the $f$-tests are different for nonsymmetric and symmetric tests.

In what follows $P_{i}, E_{i}, \operatorname{Var}_{i}$ denote the probability, expectation, and variance under $H_{i}, i=0,1$. Let $A_{\text {in }}$ and $B_{i n}^{2}$ stand for the asymptotic values of $n^{-1} E_{i} R_{n}$ and $n^{-1} \operatorname{Var}_{i} R_{n}$, receptively, and $x_{n}=\sqrt{n}\left(A_{1 n}-A_{0 n}\right) / B_{0 n}$. We briefly outline here the scheme of asymptotic study of $\omega$-IAE, the $\beta$-IAE is analyzed similarly. Performance of statistic $R_{n}$ will be measured by the asymptotic value of

$$
e_{n}^{\omega}(f)=-\log P_{0}\left\{R_{n}>n A_{1 n}\right\}=-\log P_{0}\left\{\frac{R_{n}-n A_{0 n}}{\sqrt{n} B_{0 n}}>x_{n}\right\}
$$

Nonsymmetric tests. It is known by Holst and Rao [6] that the nonsymmetric tests discriminate alternatives $H_{1}(4.1)$ with $\delta(n)=n^{-1 / 2}$, and asymptotically optimal test in Pitman's sense is a linear test with test statistic $L_{n}=\sum_{k=1}^{n} l\left(r_{k n}\right) W_{k n}$. The intermediate alternatives $H_{1}$ determine $\delta(n)$ such that

$$
\delta(n) \longrightarrow 0, \quad \sqrt{n} \delta(n) \longrightarrow \infty
$$

Denote $\rho(f) \equiv\left(1 / \sigma_{0}\right) \int_{0}^{1} l(u) \operatorname{cov}(f(Y, u), Y) d u=\int_{0}^{1} l(u) \operatorname{corr}(f(Y, u), Y) d u$. It can be shown that $x_{n}=-\sqrt{n} \delta(n) \rho(f)=o(\sqrt{n})$. Therefore, $e_{n}^{\omega}$ is determined by the asymptotic behavior of large deviation probabilities for $R_{n}$ under $H_{0}$, which is given by Theorems 2.1 and 2.2. In agreement with Kallenberg [9], this efficiency will be called weak $\omega$-IAE when $\sqrt{n} \delta(n)=O(\sqrt{\ln n})$, when $\sqrt{n} \delta(n)=o\left(n^{1 / 6}\right)$, we say middle $\omega$-IAE, and we speak of strong $\omega$-IAE when $\sqrt{n} \delta(n)=o(\sqrt{n})$. Applying Theorems 2.1 and 2.2, we get the following theorem.

Theorem 4.1. Let alternative $H_{1}$ be specified by (4.1) and (4.4). If

(i) $\int_{0}^{1} E|f(Y, u)|^{2+\varepsilon} d u<\infty$, for some $\varepsilon>0$ and $\sqrt{n} \delta(n)=O(\sqrt{\log n})$,

or

(ii) $\int_{0}^{1} E \exp \{H|f(Y, u)|\} d u<\infty$ for some $H>0$, 
then

$$
\frac{e_{n}^{\omega}(f)}{n \delta^{2}(n)}=\frac{1}{2} \rho^{2}(f)(1+o(1))
$$

It follows from Theorem 4.1 that $\rho^{2}(f)$ can be taken as a measure in sense of $\omega$-IAE of the $f$-test. Obviously $|\rho(f)| \leq\left(\int_{0}^{1} l^{2}(u) d u\right)^{1 / 2}$ and equality is achieved if and only if $f(y, u)=l(u) y$. Thus, within the class of nonsymmetric tests, the linear test (based on statistics $L_{n}$ ) is most efficient in the of $\omega$-IAE (all three types) as it was in Pitman's sense, since it satisfies condition (ii) of this theorem.

As follows from Theorem 4.1, nonsymmetric tests are "thin directed" in sense that each nonsymmetric $f$-test essentially depends on alternative $H_{1}$. Note that for linear test, $\rho^{2}(f)=\int_{0}^{1} l^{2}(u) d u$.

Symmetric tests. Symmetric tests cannot distinguish alternative $H_{1}(4.1)$ that is at a "distance" $\delta(n)=n^{-1 / 2}$, and can distinguish more distant (4.1) alternatives with $\delta(n)=n^{-1 / 4}$. Moreover within the class of symmetric tests, the optimal test in Pitman's sense is Greenwood's test based on statistics: $G_{n}=W_{1 n}^{2}+W_{2 n}^{2}+\cdots+W_{n n}^{2}$, see Holst and Rao [6]. For symmetric tests, intermediate alternatives $H_{1}$ determine $\delta(n)$ such that

$$
\delta(n) \longrightarrow 0, \quad \sqrt[4]{n} \delta(n) \longrightarrow \infty
$$

This efficiency will be weak $\omega$-IAE when $\sqrt[4]{n} \delta(n)=O(\sqrt[4]{\log n})$, when $\sqrt[4]{n} \delta(n)=o\left(n^{1 / 12}\right)$, it will be $\omega$-IAE, and strong $\omega$-IAE when $\sqrt[4]{n} \delta(n)=o(\sqrt[4]{n})$.

Put $c(f) \equiv \operatorname{corr}\left(g(Y), Y^{2}-4 y+2\right)$. Using Theorems 2.1 and 2.2, we get the following theorem.

Theorem 4.2. Let alternative $H_{1}$ be specified by (4.1) and (4.6). If (i) $E|f(Y)|^{2+\varepsilon}<\infty$, for some $\varepsilon>0$ and $\sqrt[4]{n} \delta(n)=O(\sqrt[4]{\log n})$, or (ii) $E \exp \{H|f(Y)|\}<\infty$, for some $H>0$, then

$$
\frac{e_{n}^{\omega}(f)}{\sqrt{n} \delta^{2}(n)}=\frac{1}{2} c^{2}(f)\left(\int_{0}^{1} l^{2}(u) d u\right)^{2}(1+o(1)) .
$$

The properties of the correlation coefficient imply that $c^{2}(f) \leq 1$ and $c^{2}(f)=1$ if and only if $f(y)=a y^{2}+b y+c$ (which is equivalent to $f(y)=y^{2}$ in terms of the test statistic). Thus, within the class of symmetric tests, the Greenwood statistic $G_{n}$ is most efficient in the sense of weak $\omega$-IAE (as it was in Pitman's sense) since it satisfies condition (i) of Theorem 4.2. However, for more distant alternatives when $\delta(n) \sqrt[4]{n / \log n} \rightarrow \infty, \omega$ IAE of $G_{n}$ remains an open question since this statistic does not satisfy condition (ii) of Theorem 4.2. The optimality of symmetrical tests in the sense of strong $\omega$-IAE can be deduced from Theorem 4.2 only for some subclasses of statistics satisfying Cramer's condition (ii).

Remark 4.3. The central limit theorem for $R_{n}$ implies that for $\delta(n) \sqrt[4]{n} \rightarrow \chi>0$ (i.e., for Pitman alternatives), one has $P_{0}\left\{R_{n} \geq A_{1 n}\right\}=\Phi\left(-\chi^{2} \cdot c(f) \int_{0}^{1} l^{2}(u) d u\right)$, that is, the asymptotic efficiency of test statistic $R_{n}$ is still determined by the functional $c^{2}(f)$. In other 
words, a Pitman efficient test remains optimal in the sense of $\omega$-IAE as long as Theorems 2.1 and 2.2 hold true for test statistic.

Remark 4.4. The Greenwood statistic satisfies the well-known Linnik's condition with parameter $\alpha=1 / 6$, hence relations (2.5) should be true for $x=o\left(n^{1 / 6}\right)$ (but as yet do not have a proof). Therefore, we suspect that Greenwood test must still be most efficient in middle $\omega$-IAE sense.

The proofs of Theorems 4.1 and 4.2 follow from Theorems 2.1 and 2.2 and calculations analogous to that presented by Holst and Rao [6] and Ivchenko and Mirakhmedov [7].

The analysis of $\beta$-IAE goes along the same lines. According to the general principle stated above, the efficiency of $f$-test is measured by the asymptotic value of $e_{n}^{\beta}(f)=$ $-\ln P_{1}\left(R_{n}(W)>n A_{\text {on }}\right)$. We have

$$
P_{1}\left\{R_{n}(W)<u\right\}=P_{0}\left(\tilde{R}_{n}(D)<u\right),
$$

where

$$
\widetilde{R}_{n}(D)=\sum_{m=1}^{n} \tilde{f}_{m n}\left(n D_{m n}\right), \quad \tilde{f}_{m n}(x)=f_{m n}\left(x\left(1-l\left(r_{m n}\right) \delta(n)\right)+o(\delta(n)) .\right.
$$

Omitting summand $o(\delta(n))$ for simplicity of notes and putting $z=l\left(r_{m n}\right) \delta(n)$, we get

$$
\begin{aligned}
E \exp \left\{H \tilde{f}_{m n}(Y)\right\} & =E \exp \left\{H f_{m n}(Y(1-z))\right\}=\frac{1}{1-z} \int_{0}^{\infty} \exp \left\{H f_{m n}(v)-\frac{v}{1-z}\right\} d v \\
& =\frac{1}{1-z} \int_{0}^{\infty} \exp \left\{H f_{m n}(v)-v-\frac{v z}{1-z}\right\} d v \\
& \leq \frac{1}{1-z} \int_{0}^{\infty} \exp \left\{H f_{m n}(v)-v\right\} d v \leq 2 E \exp \left\{H f_{m n}(Y)\right\}
\end{aligned}
$$

Thus we can use Theorems 2.1 and 2.2 to asymptotic analysis of $e_{n}^{\beta}(f)$. Corresponding calculation shows that for $e_{n}^{\beta}(f)$, the assertions of Theorems 4.1 and 4.2 are still true, that is, $e_{n}^{\beta}(f)$ and $e_{n}^{\omega}(f)$ are asymptotically equivalent.

\section{Appendix}

In this section, the proof of Lemmas 3.1 and 3.4 are presented. We will use the notations of Section 3. Also for notational convenience, we will write $g_{m}$ and $\tilde{g}_{m}$ instead of $g_{m}\left(Y_{m}\right)$ and $\tilde{g}_{m}\left(Y_{m}\right)$ correspondingly.

Let $F$ and $V$ be the distribution functions and $f$ and $v$ their characteristic functions correspondingly. We have, see Mirakhmedov [11],

$$
\begin{aligned}
\mid F(x) & -V(x) \mid \\
\leq & \frac{1}{\pi}\left[\int_{1 \leq|t| \leq T}|f(t)-v(t)| d t+\sup _{|t| \leq 1}\left|D_{t}(f(t)-v(t))\right|+\frac{24}{T} \max _{x}\left|D_{x} V(x)\right|\right] .
\end{aligned}
$$


14 Large deviations of the sum functions of spacings

Proof of Lemma 3.1. Put $u=i t \sigma_{n}^{-1}+h, h=x / \sigma_{n}, \tilde{\psi}_{m n}(u, \tau)=E \exp \left\{u \tilde{g}_{m}+i \tau\left(Y_{m}-1\right) n^{-1 / 2}\right\}$,

$$
\widetilde{\Psi}_{n}(u, \tau)=\prod_{m=1}^{n} \tilde{\psi}_{m n}(u, \tau), \quad \widetilde{Q}(t, \tau, x)=i t x+\frac{x^{2}}{2}-\frac{t^{2}}{2}-\frac{\tau^{2}}{2} .
$$

We have $e^{i t} \tilde{\psi}_{m n}(u, \tau \sqrt{n})=E \exp \left\{u \tilde{g}_{m}+i t Y_{m}\right\}=\int_{0}^{\infty} e^{i t \tau}\left[e^{-y} \exp \left\{u \tilde{g}_{m}(y)\right\} 1\{y \geq 0\}\right] d y$. So $e^{i t \tau} \widetilde{\psi}_{m n}(u, \tau \sqrt{n})$ is the Fourier transformation of the complex function $k(y)=$ $\left[e^{-y} E \exp \left\{u \tilde{g}_{m}(y)\right\} 1\{y \geq 0\}\right]$, and $k(y)$ and $|k(y)|^{2}$ are integrable functions. Recall the definition of $\tilde{g}_{m}$ and that $0 \leq x \leq \sqrt{\delta \ln n}$ to get $\left|E \exp \left\{u \widetilde{g}_{m}\right\}\right| \leq E \exp \left\{h \tilde{g}_{m}\right\} \leq n^{\varepsilon \delta}$. Therefore by Plansherel's identity,

$$
\int_{-\infty}^{\infty}\left|e^{i \tau} \widetilde{\psi}_{m n}(u, \tau \sqrt{n})\right|^{2} d \tau=2 \pi \int_{-\infty}^{\infty}|k(y)|^{2} d y \leq 2 \pi n^{2 \varepsilon \delta} .
$$

From this using Holder's inequality, we see that

$$
\int_{-\infty}^{\infty}\left|\tilde{\Psi}_{n}(t, \tau)\right| d \tau \leq 2 \pi n^{2 \varepsilon \delta+0.5} .
$$

Hence we can use formula (1.2) to get

$$
\tilde{\varphi}_{n}(u)=\frac{1}{2 \pi \sqrt{n} p_{n}(n, 1)} \int_{-\infty}^{\infty} \tilde{\Psi}_{n}(u, \tau) d \tau .
$$

Putting $\widetilde{\varphi}_{n}(t, h) \stackrel{\text { def }}{=} \operatorname{Eexp}\left\{i t \widetilde{G}_{n}^{*}\right\}$, we obtain

$$
\tilde{\varphi}_{n}(t, h)=\frac{\exp \{-i t x\} \tilde{\varphi}_{n}(u)}{\tilde{\varphi}_{n}(h)} .
$$

Let $a_{n}=C_{1} n^{\delta^{\prime} / 3\left(2+\delta^{\prime}\right)}, b_{n}=C_{2} n^{1 / 2-\varepsilon \delta / \delta^{\prime}}$. From (A.5), we have

$$
\begin{aligned}
\exp \{- & i t x\} \tilde{\varphi}_{n}(u) \\
= & \frac{1}{2 \pi \sqrt{n} p_{n}(n, 1)} \\
& \times\left[\int_{-\infty}^{+\infty} \exp \{-i t x+\widetilde{Q}(t, \tau, x)\} d \tau+\int_{|\tau| \leq a_{n}} e^{-i t x}\left(\widetilde{\Psi}_{n}(u, \tau)-e^{\widetilde{Q}(t, \tau, x)}\right) d \tau\right. \\
& \left.\quad+\int_{a_{n} \leq|\tau| \leq b_{n}} e^{-i t x} \widetilde{\Psi}_{n}(u, \tau) d \tau+\int_{b_{n} \leq|\tau|} e^{-i t x} \widetilde{\Psi}_{n}(u, \tau) d \tau-\int_{a_{n} \leq|\tau|} e^{-i t x+\widetilde{Q}(t, \tau, x)} d \tau\right] \\
\equiv & \frac{1}{2 \pi \sqrt{n} p_{n}(n, 1)}\left[\sum_{j=1}^{5} B_{j}\right],
\end{aligned}
$$

where $B_{j}$ denotes the $j$ th summand inside of square brackets. It is obvious that

$$
B_{1}=\sqrt{2 \pi} \exp \left\{\frac{x^{2}}{2}-\frac{t^{2}}{2}\right\}
$$

Estimation of $B_{2}$ and $B_{3}$ rests on the following lemma. 
Lemma A.5. Under conditions of Lemma 3.1, there exist constants $C_{1}, C_{2}$ such that $C_{1}<C_{2}$ and for $j=0,1$ the following assertions are true:

(1) if $|t| \leq a_{n},|\tau| \leq a_{n}^{\prime}$, then

$$
\begin{aligned}
& D_{t}^{j} \tilde{\Psi}_{n}(t, \tau)=(-t+i x)^{j} \exp \{\tilde{Q}(t, \tau, x)\}\left(1+O\left((x+|t|+|\tau|)^{2+\delta^{\prime}} n^{-\delta^{\prime} / 2+\varepsilon \delta}\right)\right), \\
& \text { where } a_{n}^{\prime}=C_{2} a_{n} / C_{1} ; \\
& \text { (2) if }|t| \leq a_{n}, a_{n}^{\prime} \leq|\tau| \leq b_{n} \text {, then }
\end{aligned}
$$

$$
\left|D_{t}^{j} \tilde{\Psi}_{n}(u, \tau)\right| \leq C \exp \left\{\frac{x^{2}}{2}-\frac{t^{2}}{2}-\frac{\tau^{2}}{4}\right\}\left(|t|+|\tau|+h n^{\varepsilon \delta}\right)^{j} .
$$

The proof of Lemma A.5 is alike to the proof of Lemma 2 from [7], and we omit it. Using Lemma A.5 with $j=0$ after simple and quite clear calculations choosing $\varepsilon<\delta^{\prime} / 4 \delta$, we obtain

$$
B_{2}+B_{3}=\exp \left\{\frac{x^{2}-t^{2}}{2}\right\}\left(1+O\left(\left(x+t^{2+\delta^{\prime}}\right) n^{-\left(\delta^{\prime} / 4\right)}\right)\right)
$$

Let now $|t| \leq a_{n},|\tau|>b_{n}$. Using successively inequalities $x<\exp \{x-1\}$ and $x-1<$ $0.5\left(x^{2}-1\right)$, we have

$$
\begin{aligned}
\left|\tilde{\psi}_{m n}(u, \tau)\right| & \leq\left|E \exp \left\{i \tau \bar{Y}_{m}\right\}\left(\exp \left\{i u \tilde{g}_{n}\right\}-1\right)+E \exp \left\{i \tau \bar{Y}_{m}\right\}\right| \leq\left|E \exp \left\{i \tau \bar{Y}_{m}\right\}\right|+|u| E\left|\tilde{g}_{m}\right| \\
& \leq \exp \left\{-\left(1-\left|E \exp \left\{i \tau \bar{Y}_{m}\right\}\right|\right)+|u| E\left|\tilde{g}_{m}\right|\right\} \\
& \leq \exp \left\{-\frac{1}{2}\left(1-\left|E \exp \left\{i \tau \bar{Y}_{m}\right\}\right|^{2}\right)+\left(|t| \sigma_{n}^{-1}+h\right) E\left|\tilde{g}_{m}\right|\right\}
\end{aligned}
$$

since $u=t \sigma_{n}^{-1}+h$. By (3.11) for $|\tau|>b_{n}$ and $n \geq C_{2}^{\delta^{\prime} / \varepsilon \delta}$, we get

$$
1-\left|E \exp \left\{i \tau \bar{Y}_{m}\right\}\right|^{2} \geq 0.5 C_{2} n^{-2 \varepsilon \delta / \delta^{\prime}} .
$$

Using Holder's inequality, we see that $\sum_{m=1}^{n} E\left|\tilde{g}_{m}\right| \leq \sigma_{n} \sqrt{n}$. Therefore for $|t| \leq a_{n}$,

$$
\frac{|t|}{\sigma_{n}} \sum_{m=1}^{n} E\left|\tilde{g}_{m}\right| \leq C_{1} n^{1 / 2+\delta^{\prime} / 3\left(2+\delta^{\prime}\right)}, \quad h \sum_{m=1}^{n} E\left|\widetilde{g}_{m}\right| \leq \delta \sqrt{n} \ln n .
$$

From (A.12), (A.13), (A.14), it follows that for any integer $k$ and $s$ such that $1 \leq k, s \leq n$ and $n \geq C_{2}^{\delta^{\prime} / \varepsilon \delta}$,

$$
\prod_{m=1, m \neq k, s}^{n}\left|\tilde{\psi}_{m n}(u, \tau)\right| \leq \exp \left\{-\frac{C_{2}}{2}(n-2) n^{-2 \varepsilon \delta / \delta^{\prime}}+C_{1} n^{1 / 2+\delta^{\prime} / 3\left(2+\delta^{\prime}\right)}+\delta \sqrt{n} \ln n\right\} .
$$

Choose here $\varepsilon<\delta^{\prime} / 6 \delta$, and $C_{2}>2 C_{1}$ to get

$$
\prod_{m=1, m \neq k, s}^{n}\left|\tilde{\psi}_{m n}(u, \tau)\right| \leq C_{3} \exp \left\{-C_{4} n^{2 / 3}\right\} .
$$


16 Large deviations of the sum functions of spacings

Using Holder's inequality and (A.3), we have

$$
\int_{-\infty}^{\infty}\left|\tilde{\psi}_{s n}(u, \tau) \tilde{\psi}_{k n}(u, \tau)\right| d \tau \leq C n^{\varepsilon \delta+0,5}
$$

From this and (A.16) for $B_{4}$, we obtain

$$
\left|B_{4}\right| \leq C \exp \left\{-c n^{2 / 3}\right\}
$$

It is easy to see that

$$
\left|B_{5}\right| \leq C \exp \left\{\frac{x^{2}}{2}-\frac{t^{2}}{2}-\frac{a_{n}^{2}}{2}\right\}
$$

Substituting relations (A.8), (A.11), (A.18), (A.19) into (A.7), we get that if $|t| \leq$ $C_{1} n^{\delta^{\prime} / 3\left(2+\delta^{\prime}\right)}$, then

$$
\exp \{-i t x\} \tilde{\varphi}_{n}(u)=\frac{\exp \left\{\left(x^{2}-t^{2}\right) / 2\right\}\left(1+O\left(n^{-\delta^{\prime} / 4}\right)\right)}{\sqrt{2 \pi n} p_{n}(n, 1)}
$$

Particularly, at $t=0$ from (A.20) and (3.11), we have

$$
\tilde{\varphi}_{n}(h)=\frac{\exp \left\{x^{2} / 2\right\}\left(1+O\left(n^{-\delta^{\prime} / 4}\right)\right)}{\sqrt{2 \pi n} p_{n}(n, 1)}=\exp \left\{\frac{x^{2}}{2}\right\}\left(1+O\left(n^{-\delta^{\prime} / 4}\right)\right) .
$$

The second assertion of Lemma 3.1 is proved.

Since (A.6) from (A.20) and (A.21), we obtain

$$
\tilde{\varphi}_{n}(t, h)=\exp \left\{\frac{-t^{2}}{2}\right\}\left(1+O\left(n^{-\delta^{\prime} / 4}\right)\right)
$$

From (A.5), (A.20), (A.21), it follows that

$$
\begin{aligned}
\frac{d}{d t} \tilde{\varphi}_{n}(u, h) & =\frac{\exp \{-i t x\}}{\tilde{\varphi}_{n}(h)}\left[\frac{d}{d t} \tilde{\varphi}_{n}(u)-i t \tilde{\varphi}_{n}(u)\right] \\
& =\frac{1}{\sqrt{2 \pi}} \exp \left\{-i t x-\frac{x^{2}}{2}\right\}\left(1+O\left(n^{-\delta^{\prime} / 4}\right)\right) \int_{-\infty}^{\infty}\left[\frac{d}{d t} \tilde{\Psi}_{n}(u, \tau)-i x \widetilde{\Psi}_{n}(u, \tau)\right] d \tau \\
& =\frac{1}{\sqrt{2 \pi}} \exp \left\{-i t x-\frac{x^{2}}{2}\right\}\left(1+O\left(n^{-\delta^{\prime} / 4}\right)\right)\left[J_{1}+J_{2}\right]
\end{aligned}
$$

where

$$
J_{1}=\int_{|\tau| \leq a_{n}}\left[\frac{d}{d t} \widetilde{\Psi}_{n}(u, \tau)-i x \widetilde{\Psi}_{n}(u, \tau)\right] d \tau, \quad J_{2}=\int_{|\tau|>a_{n}}\left[\frac{d}{d t} \widetilde{\Psi}_{n}(u, \tau)-i x \widetilde{\Psi}_{n}(u, \tau)\right] d \tau .
$$


We have

$$
\begin{aligned}
J_{1}= & \int_{|\tau| \leq a_{n}} \frac{d}{d t}\left(\widetilde{\Psi}_{n}(u, \tau)-\exp \{\widetilde{Q}(t, \tau, x)\}\right) d \tau-i x \int_{|\tau| \leq a_{n}}\left(\widetilde{\Psi}_{n}(u, \tau)-\exp \{\widetilde{Q}(t, \tau, x)\}\right) d \tau \\
& +\int_{|\tau| \leq a_{n}}\left(\frac{d}{d t} \exp \{\widetilde{Q}(t, \tau, x)\}-i x \exp \{\widetilde{Q}(t, \tau, x)\}\right) d \tau .
\end{aligned}
$$

Hence using first assertion of Lemma A.5 with $j=1$, we obtain

$$
\frac{1}{\sqrt{2 \pi}} \exp \left\{-i t x-\frac{x^{2}}{2}\right\} J_{1}=-t \exp \left\{-\frac{t^{2}}{2}\right\}+\exp \left\{-\frac{t^{2}}{2}\right\}\left(1+O\left((x+|t|)^{2+\delta^{\prime}} n^{-\delta^{\prime} / 2+\varepsilon \delta}\right)\right),
$$

because

$$
\begin{aligned}
\frac{1}{\sqrt{2 \pi}} & \exp \left\{-i t x-\frac{x^{2}}{2}\right\} \int_{|\tau| \leq a_{n}}\left(\frac{d}{d t} \exp \{\tilde{Q}(t, \tau, x)\}-i x \exp \{\widetilde{Q}(t, \tau, x)\}\right) d \tau \\
& =-t \exp \left\{-\frac{t^{2}}{2}\right\}\left(1+\frac{1}{\sqrt{2 \pi}} \int_{|\tau|>a_{n}} e^{-\tau^{2} / 2} d \tau\right)
\end{aligned}
$$

Since $n>2$, there are integers $k, s$ such that $1 \leq k, s \leq n, k \neq j, s \neq j$,

$$
\begin{aligned}
\int_{|\tau|>a_{n}} & \left|\frac{d}{d t} \widetilde{\Psi}_{n}(u, \tau) d \tau\right| \\
\leq & \sum_{j=1}^{n} \int_{|\tau|>a_{n}}\left|\frac{d}{d t} \widetilde{\psi}_{j}(u, \tau)\right| \prod_{m=1, m \neq j}^{n}\left|\widetilde{\psi}_{m}(u, \tau)\right| d \tau \\
\leq & \frac{1}{\sigma_{n}} \sum_{j=1}^{n} E\left|\tilde{g}_{j}\right| \\
& \times\left[\int_{a_{n}<|\tau|<b_{n}} \prod_{m=1, m \neq j}^{n}\left|\widetilde{\psi}_{m}(u, \tau)\right| d \tau+\int_{|\tau|>b_{n}}\left|\widetilde{\psi}_{k}(u, \tau) \tilde{\psi}_{s}(u, \tau)\right| \prod_{m=1, m \neq j, k, s}^{n}\left|\tilde{\psi}_{m}(u, \tau)\right| d \tau\right] .
\end{aligned}
$$

Therefore, using second assertion of Lemma A.5 for first integral inside of brackets, and (A.16) and (A.17) for second integral, and that $\sum_{m=1}^{n} E\left|\tilde{g}_{m}\right| \leq \sigma_{n} \sqrt{n}$, we get

$$
\int_{|\tau|>a_{n}}\left|\frac{d}{d t} \widetilde{\Psi}_{n}(u, t)\right| d \tau \leq C n \exp \left\{-c n^{2 / 3}\right\}
$$

Analogousy using second assertion of Lemma A.5, (A.16), and (A.17), we get

$$
\int_{|\tau|>a_{n}}\left|\tilde{\Psi}_{n}(u, \tau)\right| d \tau \leq C n^{0.5+\varepsilon \delta} \exp \left\{-n^{2 / 3}\right\} .
$$

Thus

$$
\left|J_{2}\right| \leq C \exp \left\{-c n^{1 / 3}\right\}
$$


Now, from (A.23), (A.26), and (A.31), it follows that

$$
\frac{d}{d t} \widetilde{\varphi}_{n}(u, h)=-t \exp \left\{-\frac{t^{2}}{2}\right\}+\exp \left\{-\frac{t^{2}}{2}\right\}\left(1+O\left((x+|t|)^{2+\delta^{\prime}} n^{-\delta^{\prime} / 2+\varepsilon \delta}\right)+C \theta \exp \left\{-c n^{1 / 3}\right\} .\right.
$$

Putting in (A.1) $F(x)=P\left(\widetilde{G}_{n}^{*}<x\right), V(x)=\Phi(x), f(t)=\tilde{\varphi}_{n}(t, h), v(t)=\exp \left\{-t^{2} / 2\right\}$, and $T=n^{\delta^{\prime} / 3\left(2+\delta^{\prime}\right)}$, and using (A.22) and (A.32), we complete the proof of Lemma 3.1.

Proof of Lemma 3.4. Recall that $\lambda$ and $h$ are the roots of (3.5) and (3.11) correspondingly. Put $u=i t \mu^{-1}(h, \lambda)+h, \varphi_{n}(t, h)=E \exp \left\{i t G_{n}^{*}(h, \lambda)\right\}, \psi_{m}(u, \tau)=K_{m}\left(u, \tau n^{-1 / 2}, \lambda\right)=$ $E \exp \left\{u g_{m}\left(Y_{m}(\lambda)\right)+i \tau\left(Y_{m}(\lambda)-1\right) n^{-1 / 2}\right\}, \Psi_{n}(u, \tau)=\prod_{m=1}^{n} \psi_{m}(u, \tau)$.

Lemma A.6. Under conditions of the Theorem 2.2, there exist $C_{0}, C_{1}, C_{2}$, such that if $|t| \leq$ $C_{1} n^{1 / 2}$, then for sufficiently large $n$ and $j=0,1$,

$$
\left|\frac{d^{j}}{d t^{j}}\left(\varphi_{n}(t, h)-\exp \left\{-\frac{t^{2}}{2}\right\}\right)\right| \leq C_{2}\left(1+|t|^{3}\right) n^{-1 / 2} \exp \left\{-C_{0} t^{2}\right\}
$$

Proof. Recalling that $\varphi_{n}(z)=E \exp \left\{z T_{n}(D)\right\}$, we get

$$
\varphi_{n}(t, h)=\frac{\varphi_{n}(u)}{\varphi_{n}(h)} \exp \left\{-\frac{i t A(h, \lambda)}{\mu(h, \lambda)}\right\}
$$

Alike (A.3), now we have

$$
\int_{-\infty}^{\infty}\left|\psi_{m}(u, \tau \sqrt{n})\right|^{2} d \tau \leq \pi \lambda\left(\frac{C_{0}}{1-2\left(H_{1}+\varepsilon\right)}\right)^{1 / 2}
$$

by (3.13). Hence using Holder's inequality, we see that

$$
\int_{-\infty}^{\infty}\left|\Psi_{n}(t, \tau)\right| d \tau \leq 2 \pi\left(\sqrt{\frac{n C_{0}}{1-2\left(H_{1}+\varepsilon\right)}}\right)^{1 / 2} .
$$

Therefore from (1.2),

$$
\varphi_{n}(u)=\frac{1}{2 \pi \sqrt{n} p_{n}(n, \lambda)} \int_{-\infty}^{\infty} \Psi_{n}(u, \tau) d \tau=\frac{1}{2 \pi \sqrt{n} p_{n}(n, \lambda)}\left[\mathfrak{I}_{1}+\mathfrak{I}_{2}\right]
$$

where

$$
\mathfrak{J}_{1}=\int_{|\tau| \leq C_{2} \sqrt{n}} \Psi_{n}(u, \tau) d \tau, \quad \mathfrak{I}_{2}=\int_{|\tau|>C_{2} \sqrt{n}} \Psi_{n}(u, \tau) d \tau .
$$

Let $|t| \leq C_{1} n^{1 / 2}$ and $|\tau| \leq C_{3} n^{1 / 2}$, where $C_{1}$ and $C_{3}$ do not exceed $H_{2}$. Then by (3.15), $\left|\psi_{m}(u, \tau)\right| \geq 1 / 2$. Hence for $\ln \psi_{m}(u, \tau)$, Taylor's expansion formula at point $(h, 0)$ can be used. This expansion together with Lemma 3.2 and representation of $A(h, \lambda), B^{2}(h, \lambda)$, 
$\sigma^{2}(h, \lambda)$ and $\rho(h, \lambda)$ as in the first equalities in (3.28), (3.30), (3.31), and (3.32), respectively, and that $\mu^{2}(h, \lambda)=\sigma^{2}(h, \lambda)-\rho^{2}(h, \lambda) B^{-2}(h, \lambda)$ after simple manipulations give the following relation:

$$
\begin{aligned}
\sum_{m=1}^{n} \ln \psi_{m}(u, \tau)= & \sum_{m=}^{n} \ln \psi_{m}(h, 0)+\frac{i t A(h, \lambda)}{\mu(h, \lambda)}+\frac{i \tau \Lambda(h, \lambda)}{\sqrt{n}}-\frac{t^{2} \sigma^{2}(h, \lambda)}{2 \mu^{2}(h, \lambda)}-\frac{\tau t \rho(h, \lambda)}{\mu(h, \lambda) \sqrt{n}} \\
& -\frac{\tau^{2} B^{2}(h, \lambda)}{2 n}+O\left(\frac{t^{3}+\tau^{3}}{\sqrt{n}}\right) \\
= & \sum_{m=1}^{n} \ln \psi_{m}(h, 0)+\frac{i t A(h, \lambda)}{\mu(h, \lambda)}-\frac{t^{2}}{2} \\
& -\frac{B^{2}(h, \lambda)}{2 n}\left(\tau+t \frac{\sqrt{n} \rho(h, \lambda)}{\mu(h, \lambda) B^{2}(h, \lambda)}\right)^{2}+O\left(\frac{t^{3}+\tau^{3}}{\sqrt{n}}\right) .
\end{aligned}
$$

We note that due to condition (2.3), and relations (3.30), (3.32), and (3.33) for sufficiently large $n$, we have $\left|\sqrt{n} \rho(h, \lambda) / \mu(h, \lambda) B^{2}(h, \lambda)\right| \leq C h, B^{2}(h, \lambda) \geq n / 2$. Keeping in mind this inequalities and using (A.39) and simple manipulations alike to the proof of relation $[15,(24)]$, we get

$$
\begin{aligned}
& \left|\frac{\exp \{-i t A(h, \lambda) / \mu(h, \lambda)\}}{\Psi_{n}(h, 0)} \Psi_{n}(u, \tau)-\exp \left\{-\frac{t^{2}}{2}-\frac{B^{2}(h, \lambda)}{2 n}\left(\tau+t \frac{\sqrt{n} \rho(h, \lambda)}{\mu(h, \lambda) B^{2}(h, \lambda)}\right)^{2}\right\}\right| \\
& \leq C \frac{|t|^{3}+|\tau|^{3}}{\sqrt{n}} \exp \left\{-\frac{t^{2}}{8}-\frac{1}{8}\left(\tau+t \frac{\sqrt{n} \rho(h, \lambda)}{\mu(h, \lambda) B^{2}(h, \lambda)}\right)^{2}\right\} .
\end{aligned}
$$

Hence

$$
\left|\frac{\sqrt{n} \exp \{-i t A(h, \lambda) / \mu(h, \lambda)\}}{B(h, \lambda) \Psi_{n}(h, 0)} \mathfrak{I}_{1}-\exp \left\{-\frac{t^{2}}{2}\right\}\right| \leq C_{4} \frac{|t|^{3}+1}{\sqrt{n}} \exp \left\{-\frac{t^{2}}{16}\right\} .
$$

Let now $|t| \leq C_{1} \sqrt{n}$ and $|\tau|>C_{1} \sqrt{n}$. Alike (A.8), we have

$$
\begin{aligned}
\left|\psi_{m n}(u, \tau)\right| & \leq\left|E \exp \left\{\frac{i \tau Y_{m}(\lambda)}{\sqrt{n}}\right\}\left(\exp \left\{i u g_{m}\left(Y_{m}(\lambda)\right)\right\}-1\right)+E \exp \left\{\frac{i \tau Y_{m}(\lambda)}{\sqrt{n}}\right\}\right| \\
& \leq\left|\psi_{n}(0, \tau)\right|+|u| E\left|g_{m}\left(Y_{m}(\lambda)\right)\right| \\
& \leq \exp \left\{-\left(1-\left|\psi_{m}(0, \tau)\right|\right)+|u| E\left|g_{m}\left(Y_{m}(\lambda)\right)\right|\right\} \\
& \leq \exp \left\{-\frac{1}{2}\left(1-\left|\psi_{m}(0, \tau)\right|^{2}\right)+\left(\frac{|t|}{\sigma_{n}}+h\right) E\left|g_{m}\left(Y_{m}(\lambda)\right)\right|\right\} .
\end{aligned}
$$

Because $\left|\psi_{m}(0, \tau)\right|^{2}=\lambda^{2} /\left(\lambda^{2}+\tau^{2} / n\right)$ and (3.21), we have for sufficiently large $n, 1-$ $\left|\psi_{m}(0, \tau)\right|^{2} \geq C_{3}^{2} /\left(1+C_{3}^{2}\right) \equiv C_{5}$. Using this inequality and (3.13) in (A.42), we obtain $\left|\psi_{m}(u, \tau)\right| \leq \exp \left\{-2^{-1} C_{5}+C_{1} C_{6}\right\} \leq \exp \left\{-C_{5} / 4\right\}$, with $C_{6} \leq\left(e^{2 H} C_{0} / H_{1}^{2}\left(1-2\left(H_{1}\right.\right.\right.$ $+\varepsilon)))^{1 / 2}$ and $C_{1}<C_{4} / 4 C_{5}$. From (A.35), we see that $\int_{-\infty}^{\infty}\left|\psi_{s}(u, \tau) \psi_{k}(u, \tau)\right| d \tau \leq$ $2 \pi \sqrt{n C_{0} /\left(1-2\left(\varepsilon+H_{1}\right)\right)}, 1 \leq s, k \leq n$. 
Thus for sufficiently large $n$,

$$
\left|\mathfrak{J}_{2}\right| \leq \int_{|\tau|>C_{3} / \sqrt{n}}\left|\psi_{s}(u, \tau)\right|\left|\psi_{k}(u, \tau)\right| \prod_{m=1, m \neq k, s}^{n}\left|\psi_{m}(u, \tau)\right| d \tau \leq C_{8} \exp \left\{-C_{7} n\right\} .
$$

For sufficiently large $n$, we have $\psi_{m}(h, 0) \geq e^{-C_{6} / 2}$, and $\lambda=1+O(h)$. Therefore, it follows from (A.43) and (3.30) that for sufficiently large $n$,

$$
\left|\frac{\sqrt{n} \exp \{-i t A(h, \lambda) / \mu(h, \lambda)\}}{\lambda B(h, \lambda) \Psi_{n}(h, 0)} \mathfrak{J}_{2}\right| \leq \exp \left\{-C_{6} n\right\} .
$$

Taking into account that $|t| \leq C_{1} n^{1 / 2}$ from (A.37), (A.41), and (A.44), we obtain

$$
\begin{aligned}
& \left|\frac{\sqrt{n} \exp \{-i t A(h, \lambda) / \mu(h, \lambda)\} \sqrt{2 \pi n} p_{n}(n, \lambda)}{B(h, \lambda) \Psi_{n}(h, 0)} \varphi_{n}(u)-\exp \left\{-\frac{t^{2}}{2}\right\}\right| \\
& \quad \leq C_{7} \frac{|t|^{3}+1}{\sqrt{n}} \exp \left\{-C_{8} t^{2}\right\} .
\end{aligned}
$$

Putting $t=0$ in (A.45), taking into account (3.30), and that $\Psi_{n}(h, 0)=\prod_{m=1}^{n} K_{m}(h, 0, \lambda)$, we obtain the second assertion of Lemma 3.4. Using (A.45) and second assertion of Lemma 3.4 in the equality (A.34), we immediately get Lemma A.6 at $j=0$. From (A.34) and (1.2), we have

$$
\begin{aligned}
D_{t} \varphi_{n}(t, h)= & \varphi_{n}^{-1}(h) \exp \left\{-\frac{i t A(h, \lambda)}{\mu(h, \lambda)}\right\}\left[D_{t} \varphi_{n}(u)-\frac{i A(h, \lambda)}{\mu(h, \lambda)} \varphi_{n}(u)\right] \\
= & \frac{\exp \{-i t A(h, \lambda) / \mu(h, \lambda)\}}{\varphi_{n}(h) \sqrt{2 \pi n} p_{n}(n, \lambda)} \\
& \times\left[\int_{|\tau| \leq C_{3} \sqrt{n}} \Psi_{n}(u, \lambda)\left(\sum_{m=1}^{n} D_{t} \psi_{m}(u, \tau)-\frac{i A(h, \lambda)}{\mu(h, \lambda)}\right) d \tau\right. \\
& \left.\quad+\int_{|\tau|>C_{3} \sqrt{n}} D_{t} \Psi_{n}(u, \lambda) d \tau+\int_{|\tau|>C_{3} \sqrt{n}} \frac{i A(h, \lambda)}{\mu(h, \lambda)} \Psi_{n}(u, \lambda) d \tau\right] .
\end{aligned}
$$

Using (3.26), (3.28)-(3.33), it can be proved that

$$
\sum_{m=1}^{n} D_{t} \ln \psi_{m}(u, \tau)=\frac{i A(h, \lambda)}{\mu(h, \lambda)}-t+O\left(\tau h+\frac{t^{2}+\tau^{2}}{\sqrt{n}}\right) .
$$

It is obviously that $\left|D_{t} \psi_{m}(u, \tau)\right| \leq|t| \mu^{-1}(h, \lambda) E\left|g_{m}\right| \exp \left\{h\left|g_{m}\right|\right\}$, therefore by (3.13), (3.33), condition (2.3), we obtain alike (A.43) that

$$
\begin{aligned}
\int_{|\tau|>C_{3} \sqrt{n}}\left|D_{t} \Psi_{n}(u, \tau)\right| d \tau & \leq \sum_{l=1}^{n} \int_{|\tau|>C_{2} \sqrt{n}}\left|\psi_{s}(u, \tau) \psi_{k}(u, \tau)\right| \prod_{\substack{m=1 \\
m \neq s, k, l}}^{n}\left|\psi_{m}(u, \tau)\right|\left|D_{t} \psi_{l}(u, \tau)\right| d \tau \\
& \leq C n \exp \left\{-C_{9} n\right\} .
\end{aligned}
$$


Using in (A.46) the relations (A.40), (A.47), (A.48), (3.28), (3.33), and reasoning used at relation (A.44), we are convinced of validity of Lemma A.6 for $j=1$. Proof of Lemma A.6 is complete.

Now putting in (A.1) $F(x)=P\left\{G_{n}^{*}(h, \lambda)<x\right\}, V(x)=\Phi(x), f(t)=\varphi_{n}(t, h), T=$ $C_{1} n^{1 / 2}$, and $v(t)=\exp \left\{-t^{2} / 2\right\}$, and using Lemma A.6, we complete the proof of the first assertion of Lemma 3.4. Proof of Lemma 3.4 is complete.

\section{Acknowledgments}

I would like to express gratitude to the referee whose remarks have improved the maintenance of the present paper. I am thankful to Higher Education Commission of Pakistan under Foreign Faculty Hiring Program of which I am working in Ghulam Tshaq Khan (GIK) Institute of Engineering Sciences and Technology.

\section{References}

[1] J. Bartoszewicz, Bahadur and Hodges-Lehmann approximate efficiencies of tests based on spacings, Statistics \& Probability Letters 23 (1995), no. 3, 211-220.

[2] P. Deheuvels, Spacings and applications, Probability and Statistical Decision Theory, Vol. A (Bad Tatzmannsdorf, 1983) (F. Konecny, J. Mogyoródi, and W. Wertz, eds.), Reidel, Dordrecht, 1985, pp. 1-30.

[3] R. J. M. M. Does, R. Helmers, and C. A. J. Klaassen, On the Edgeworth expansion for the sum of a function of uniform spacings, Journal of Statistical Planning and Inference 17 (1987), no. 2, 149-157.

[4] V. Feller, An Introduction to Probability Theory and Its Applications. Vol. 2, "Mir”, Moscow, 1967.

[5] K. Ghosh and S. R. Jammalamadaka, A general estimation method using spacings, Journal of Statistical Planning and Inference 93 (2001), no. 1-2, 71-82.

[6] L. Holst and J. S. Rao, Asymptotic spacings theory with applications to the two-sample problem, The Canadian Journal of Statistics 9 (1981), no. 1, 79-89.

[7] G. I. Ivchenko and S. M. Mirakhmedov, Large deviations and intermediate efficiency of decomposable statistics in a multinomial scheme, Mathematical Methods of Statistics 4 (1995), no. 3 , 294-311.

[8] S. R. Jammalamadaka, X. Zhou, and R. C. Tiwari, Asymptotic efficiencies of spacings tests for goodness of fit, Metrika 36 (1989), no. 6, 355-377.

[9] W. C. M. Kallenberg, Intermediate efficiency, theory and examples, The Annals of Statistics 11 (1983), no. 1, 170-182.

[10] P. L'Ecuyer, Tests based on sum-functions of spacings for uniform random numbers, Journal of Statistical Computation and Simulation 59 (1997), no. 3, 251-269.

[11] S. M. Mirakhmedov, Lower estimation of the remainder term in the CLT for a sum of the functions of $k$-spacings, Statistics \& Probability Letters 73 (2005), no. 4, 411-424.

[12] V. V. Petrov, Sums of Independent Random Variables, 1st ed., Ergebnisse der Mathematik und ihrer Grenzgebiete, vol. 82, Springer, New York, 1975.

[13] R. Pyke, Spacings, Journal of the Royal Statistical Society. Series B (Methodological) 27 (1965), no. 3, 395-449.

[14] _ Spacings revisited, Proceedings of the Sixth Berkeley Symposium on Mathematical Statistics and Probability (University of California, Berkeley, Calif, 1970/1971), Vol. I: Theory of Statistics, University of California Press, California, 1972, pp. 417-427. 
[15] S. Kh. Sirazhdinov, S. M. Mirakhmedov, and Sh. A. Ismatullaev, Probabilities of large deviations for randomized decomposable statistics in a multinomial scheme, Theory of Probability and Its Applications 34 (1989), no. 4, 645-657 (1990).

[16] X. Zhou and S. R. Jammalamadaka, Bahadur efficiencies of spacings test for goodness of fit, Annals of the Institute of Statistical Mathematics 41 (1989), no. 3, 541-553.

Sherzod Mira'zam Mirakhmedov: Ghulam Ishaq Khan Institute of Engineering Sciences and Technology, Topi-23460, Swabi, N.W.F.P., Pakistan

E-mail address: shmirakhmedov@yahoo.com 


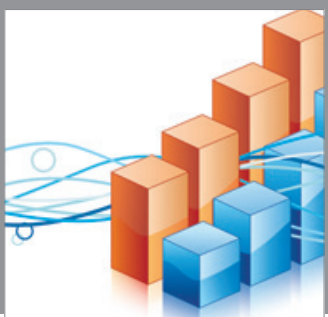

Advances in

Operations Research

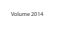

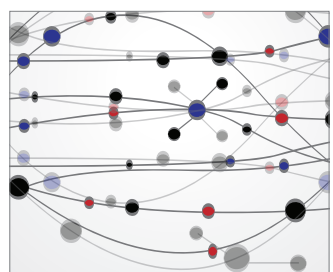

\section{The Scientific} World Journal
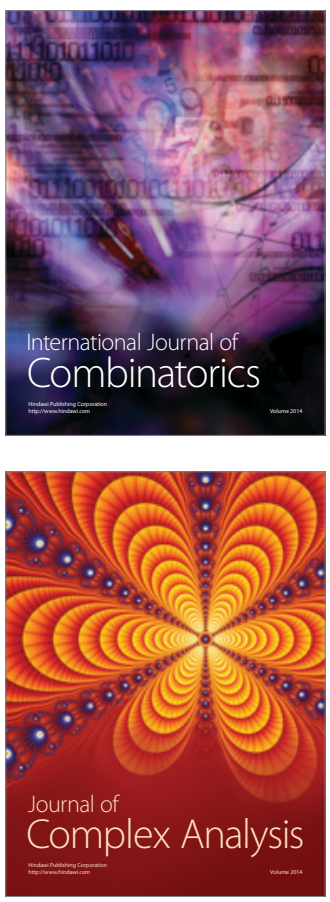

International Journal of

Mathematics and

Mathematical

Sciences
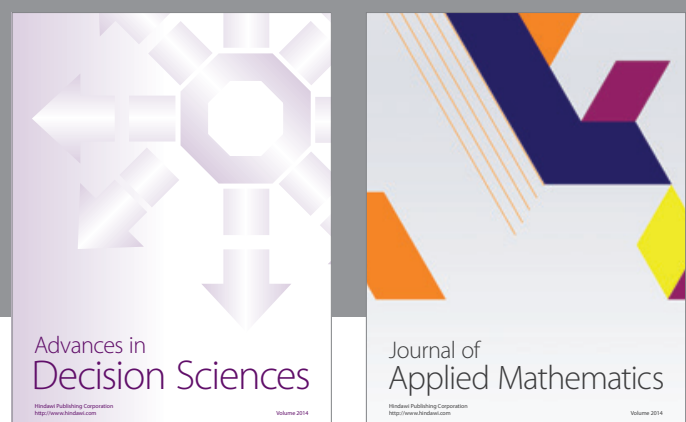

Journal of

Applied Mathematics
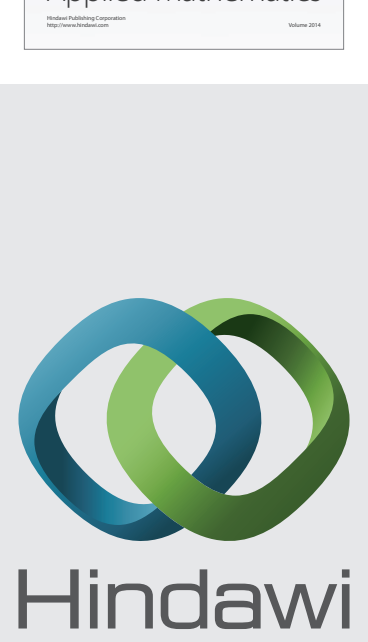

Submit your manuscripts at http://www.hindawi.com
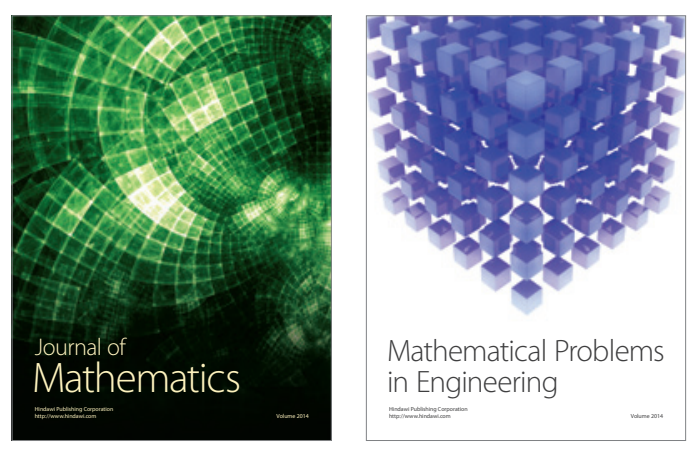

Mathematical Problems in Engineering
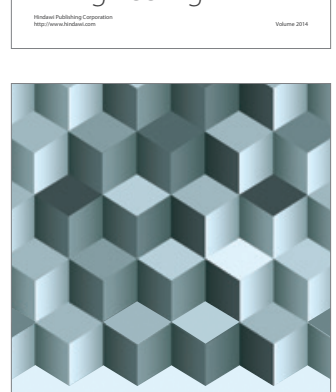

Journal of

Function Spaces
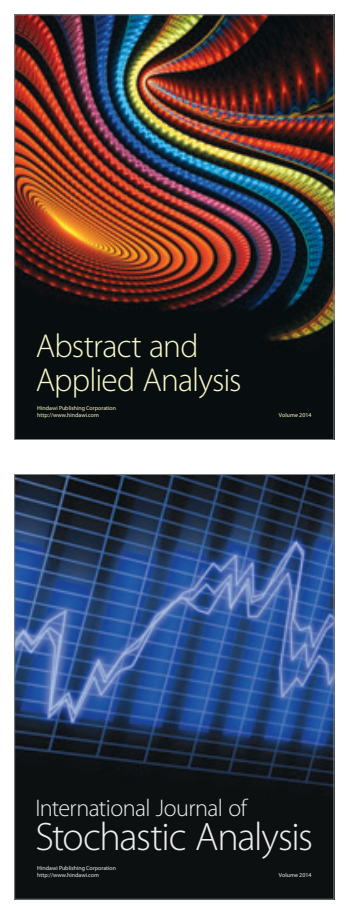

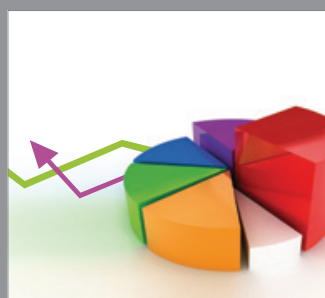

ournal of

Probability and Statistics

Promensencen
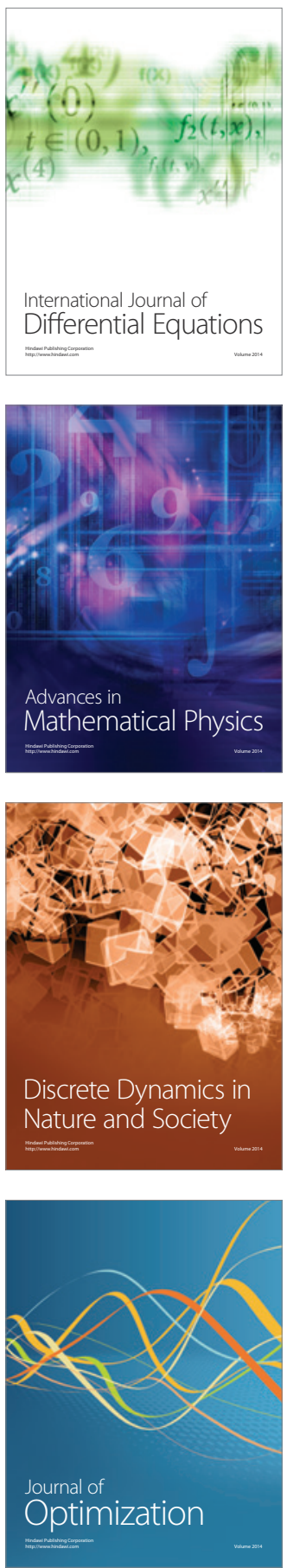